\title{
Microsensors and Actuators for Macrofluidic Control
}

\author{
Adam Huang, James Lew, Yong Xu, Yu-Chong Tai, and Chih-Ming Ho, Member, IEEE
}

\begin{abstract}
Microsensors and actuators suitable for macrofluidic control have been designed, fabricated, tested, and optimized over the span of the last decade. MEMS-based shear stress sensor arrays using polysilicon hot filaments have been fabricated on both rigid (silicon) and flexible (parylene) substrate for application on all types of fluid dynamic and aerodynamic surfaces. In addition, MEMS bubble flap-type pneumatic actuators have been tested and used in turbulent boundary layer drag reduction in conjunction with the rigid MEMS shear stress sensor arrays acting as highspeed shear stress imagers. The flexible MEMS bubble actuator arrays have also been used with the flexible shear stress sensor arrays for generating maneuvering forces in the wind tunnel for a delta wing model and on UAV-type radio-controlled aircraft.
\end{abstract}

Index Terms-Macrofluidic control, turbulent boundary layer (TBL) drag reduction, vortex shifting concept (VSC).

\section{INTRODUCTION}

A FTER a decade of intense research by the MEMS community in designing and fabricating microdevices during the 1990s, the result is the rapid growth of mature MEMS devices used by application engineers today. In the field of aerodynamics, the goal has always been the manipulation of fluidic flows with the appropriated degree of spatial and temporal resolutions required for performance enhancement. Since sensors are naturally more capable of interfacing with machineries across a large spectrum of length scales (from submicrons to meters), the adoption of microfabrication for sensors have consistently shown overwhelming technical and economical advantages compared against almost all conventional sensor technologies. Hence, today's dominant market shares of MEMS-based sensors such as accelerometers (Analog Devices, Inc.) [1], magnetometers (Honeywell) [2], and pressure sensors (Measurement Specialties, Inc.) [3]. However, the proliferation of MEMS actuators is generally limited due to scaling issues. Typically, the size of actuators needs to be within a few orders of magnitude of the object being affected. More specifically, this is due to the necessity of momentum matching between the effector (actuator) and the affected. Often times, MEMS actuators use the inherently high speeds of actuation to trade against low actuator inertia. The resulting MEMS actuators, in comparison with tradi-

\footnotetext{
Manuscript received September 2, 2003; revised March 24, 2004. This work was supported in part by the U.S. Department of Defense, Defense Advanced Projects Agency (Micro Technology Office), in part by the Air Force Office of Scientific Research (Multidisciplinary University Research Initiative), and in part by the NASA Dryden. The associate editor coordinating the review of this paper and approving it for publication was Prof. Andrei Shkel.

A. Huang, J. Lew, and C.-M. Ho are with the Mechanical and Aerospace Department, University of California, Los Angeles, CA 90095 USA (e-mail: pohao@seas.ucla.edu; lewjam@yahoo.com; chihming@seas.ucla.ed).

$\mathrm{Y} . \mathrm{Xu}$ is with the Department of Electrical and Computer Engineering, Wayne State University, Detroit, MI 48202 USA (e-mail: yxu@indigo.eng.wayne.edu).

Y.-C. Tai is with the California Institute of Technology, Pasadena, CA 91125

USA (e-mail: yctai@mems.caltech.edu).

Digital Object Identifier 10.1109/JSEN.2004.830949
}

tional actuator technologies, are typically of higher performance if the affected is inertia matched and adequate (or poorer) performance when speed versus inertia tradeoffs are made. Therefore, the two noticeable successful MEMS actuators are the inkjet printers (Hewlett Packard) [4] and Digital Micromirror Device projectors (Texas Instrument, Inc.) [5], since the affected are relatively low-inertia ink droplets and color spectrum photons, respectively.

As rationalized above, it may seem counter-intuitive to adopt MEMS technology for macrofluidic control from the perspective of serious actuator mismatch with the physical phenomenon. The primer lies in the physics of specific fluidic conditions rather than solely on the actuators. Microactuators can affect macro effects under two circumstances: 1) if the interaction effects are due to surface area or 2) if the actuation effects can propagate into global structures. These two techniques are taken to our advantage for macrofluidic control, namely the drag reduction in a turbulent boundary layer (TBL) and the aerodynamic control of a delta wing using the vortex shift concept (VSC).

\section{TBL DRAG REDUCTION}

\section{A. TBL Characteristics}

Near the surface of an aircraft, laminar boundary layers almost always develop into turbulent boundary layers. The turbulent boundary layer flow is characterized by unsteady and random flow velocity anywhere in the flow field in addition to higher velocity gradient (surface shear stress) at the wall and thicker boundary layers when compared with laminar flows. The resulting effect on an aero vehicle is increased skin friction coefficient in comparison to the laminar regions [6]. On the surface of the aircraft under the TBL are the randomly distributed high-speed streaks that have transverse length scales (along the flow) on the order of hundreds of microns and life spans on the order of few milliseconds [7]. While the exact mechanism on the creation of these streaks is still hotly debated in the fluid dynamics community, the generally accepted view is that such vortex streaks are the biggest contributors to the rise in TBL skin friction coefficient. In order to reduce this significant component of surface drag, a high-response microactuator and the corresponding microsensors must be developed to match the length scale and life span of the streaks. Fig. 1 shows the typical shear streak corresponding to the rise in local shear stress level.

\section{B. Microshear Stress Sensors for TBL}

Based on the theories of fluid dynamics, all that is necessary for knowing the forces on an aircraft (or, basically, any fluid dynamic body) are the pressure and surface shear stress distribution. However, unlike pressure sensors, shear stress sensors are not common and usually not available commercially in terms 


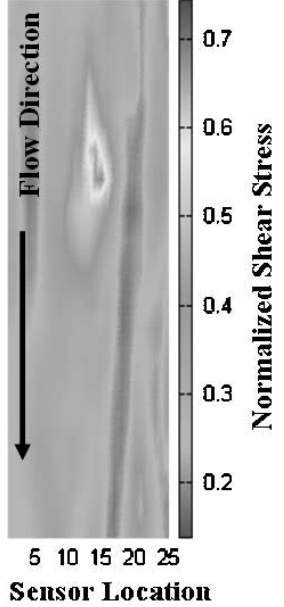

Fig. 1. Typical surface streak detected with the MEMS shear stress sensor array.

of submillimeter resolutions and kilohertz frequency responses as required to match the detection requirements for the surface streaks. Conventional transducers, such as Stanton [8], [9] and Preston [10], [11] tubes, have low-frequency responses (tens to hundreds of hertz) while being relatively large.

In terms of microshear stress sensor, there are basically two major sensing methods. One is the direct mechanical measurement of surface displacement of a tethered plate due to shear while the other is indirect measurement through thermal transport. Although there are numerous detection methods for sensing the displacement on mechanical sensors (i.e., piezoresistive [12], [13], capacitive [14], [15], and optical [16]), the common problem is the fact that such sensors have very small gaps that separate the moving surface from the anchors making it very prone to clogging by dust. Thus, for this project, the indirect method of using thermal sensing is used. The surface shear is sensed by the correlation between the convective heat transfer and the surface flow velocities. Each sensor element is simply a geometrically optimized doped-polysilicon filament that is heated to temperatures above the ambient to facilitate heat transfer (typical overheat ratios ranging from 10\%-30\%) [17]. The doped polysilicon filament with its corresponding negative temperature coefficient of resistance (TCR) allows the attainment of significantly higher response bandwidth in comparison to the typical metal filaments of hot wire anemometry (positive TCR). The filament then sits on top of a silicon nitride membrane with a vacuum chamber underneath. This significantly reduces the heat capacity surrounding the sensor relative to the ambient air, creating a high shear stress sensitive sensor [18] (typically $\sim 50 \mathrm{mV} / \mathrm{Pa}$ without amplification). The vacuum chamber is not required if high sensitivity is not needed or for operation in fluids with high heat capacities (i.e., under water). Fig. 2(a) shows the schematic of a single element of the thermal shear stress sensor. An array of such sensor elements is then distributed on the silicon wafer to create shear stress imagers [19]. Fig. 2(b) shows the MEMS shear stress imager with the magnification of the single-sensor element. The imagine zone is about $1 \times 1 \mathrm{~cm}$ and contains over 100 shear stress sensors or temperature sensors (including test sensors). Each element area is about $250 \times 250 \mu \mathrm{m}$ and the sensing hot "bridge" is

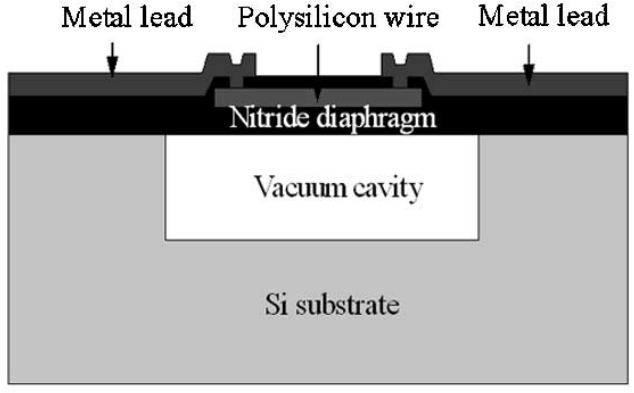

(a)

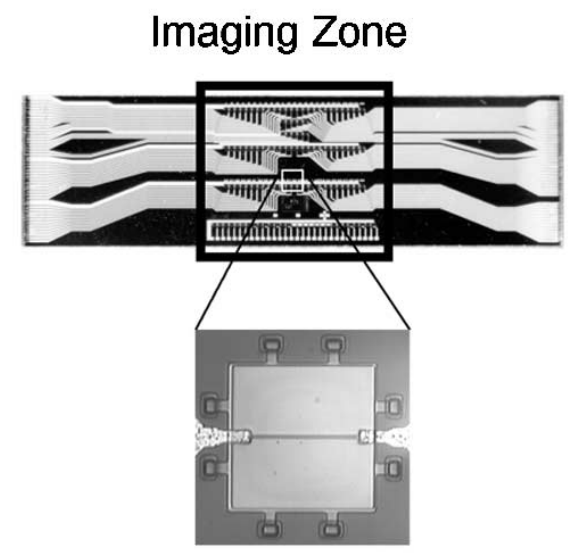

(b)

Fig. 2. (a) Schematic diagram of the thermal shear stress sensor element. (b) Micrograph of the MEMS shear stress imager chip with over 80 sensors and the corresponding single-element sensor.

a $150 \times 3 \times 0.5 \mu \mathrm{m}$ polysilicon wire. Theoretically, a much finer resolution of $<50 \times 50-\mu \mathrm{m}$ element area is possible if the interconnecting aluminum wires are more efficiently routed or by using an on-chip CMOS circuitry to distribute power and convert the signal into digital format.

One common concern with the MEMS surface shear stress sensor developed for this project is the pressure sensitivity of the sensor elements. Specifically, the sensor element of doped polysilicon (piezoelectric) on top of a thin diaphragm closely resembles a typical MEMS pressure sensor. This is a practical concern that is application relevant. For example, the MEMS shear stress sensor used for the TBL experiments would present a problem if used in experiments with significant pressure variation (calibrations against pressure must be used). Thus, for underwater applications, a change of sensor element geometries in addition to the protective parylene overcoat is needed to reduce the pressure sensitivity [20]. Instead of such drastic design changes, it may be easier to simply forgo the vacuum cavity since liquid mediums have much higher thermal capacities that will increase sensor sensitivity. This may be a more practical tradeoff since, even with design changes, pressure sensitivity of the sensor is merely reduced, but not eliminated. In terms of the TBL experiment, pressure sensitivity should not be a concern. It has been shown that the peak pressure fluctuation intensity of the separating turbulent boundary layer is only a few percentage of the dynamic pressure of the freestream [21]. In the TBL experiment, the maximum freestream velocity tested at $17 \mathrm{~m} / \mathrm{s}$ corresponds to $178 \mathrm{~Pa}$ of dynamic pressure. The maximum flap tip velocity is only on the order of $0.01-0.1 \mathrm{~m} / \mathrm{s}$. Fig. 3 shows the 


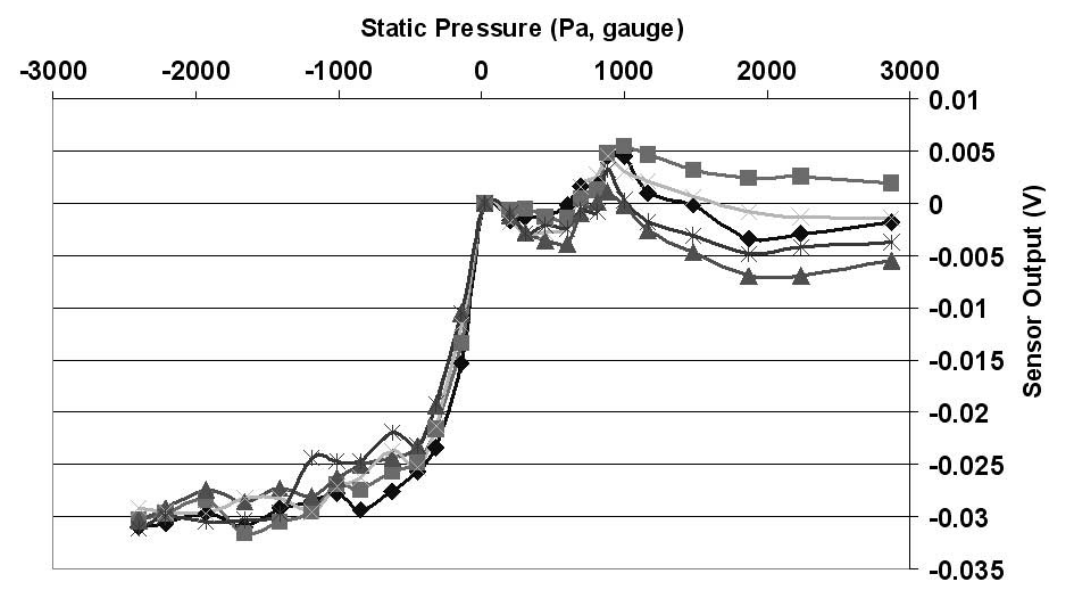

Fig. 3. Pressure sensitivity of the MEMS shear stress sensor. Pressure measured relative to the ambient.

pressure dependence of five sensors from the same shear stress sensor array used for the TBL experiments. Notice that the test range is significantly greater than the maximum dynamic pressure case. Also note that the positive pressure dependence is not very consistent, as reported in [20], while the negative pressure dependence is relatively large for the first $1000 \mathrm{~Pa}$. In any event, the typical peak shear stress reduction detected is in the order of $0.2 \mathrm{~Pa}$ or higher (with $17 \mathrm{~m} / \mathrm{s}$ freestream velocity), which corresponds to $\sim 100-\mathrm{mV}$ sensor output. Characterizations performed by other fluid dynamic researchers such as Ruedi et al. [22] have also noted a lack of significant pressure dependence specific to this type of applications. For other characterizations of the MEMS surface shear stress sensors, Österlund et al. [23] should be consulted by interested readers.

\section{Microballoon-Flap Actuators for TBL}

It is fair to say that, even today large force, displacement, and high-step response actuators of $\sim 10-100 \mathrm{mN},>100 \mu \mathrm{m}$, and $>100 \mathrm{~Hz}$, respectively, are hard to come by. The actuator required for turbulent boundary layer drag reduction needs to be no larger than a few millimeters in size and have step response faster than $10 \mathrm{~ms}$. Initially, magnetic [24], [25] (Fig. 4), or electromagnetic [26] based, actuators were used. The magnetic flap consists of electroplated $\mathrm{Ni}_{80} \mathrm{Fe}_{20}$ alloy hinged on a torsion bar. The electromagnetic flap actuator replaced the $\mathrm{Ni}_{80} \mathrm{Fe}_{20}$ with patterned copper coils. In both cases, various actuator sizes on the order of millimeter dimensions were fabricated and the actuation scheme involves the use of a permanent magnet on the back side of the actuator. Both methods have very significant drawbacks. The magnetic flap actuator cannot be actuated with high speeds due to the necessity of moving the permanent magnet to bring the corresponding actuation. The electromagnetic flap actuator can actuate at high speeds, but this is only possible at the first-order actuator resonant frequency of $\sim 1.3 \mathrm{kHz}$ to reach sufficient amplitudes of $>100 \mu \mathrm{m}$. Due to the nature of the random distribution of the shear streaks, the step response, not the resonant response, needs to match with the streak life span.

The next actuator used is the microballoon actuator [27]. This consists of a layer of spun on RTV silicone rubber with a pneumatic manifold underneath for pressurized air actuation. The

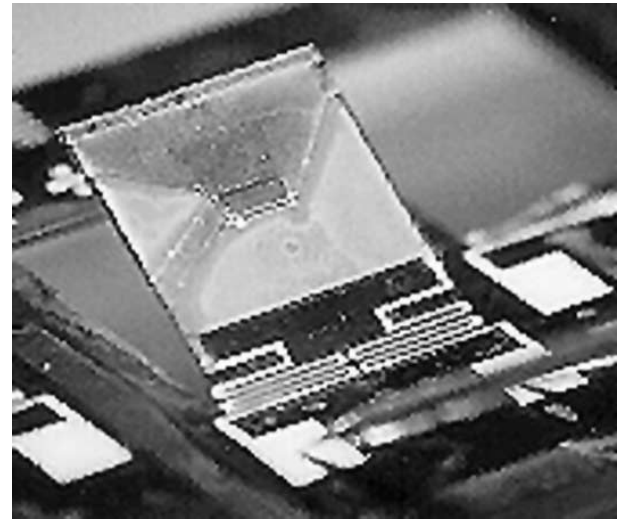

Fig. 4. $\mathrm{Ni}_{80} \mathrm{Fe}_{20}$, permalloy, magnetic flap. The electromagnetic flap version is similar, except with the addition of patterned copper coils on the flap.

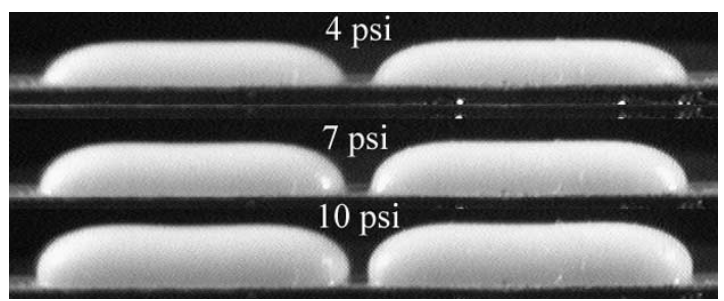

Fig. 5. Microballoon actuator actuation height versus drive pressure. 10-psi actuation corresponds to 2-mm out-of-plane actuation.

actuator is simply the deformed shape of the silicone rubber activated by pressurizing and decompressing via an off-board miniature solenoid valve. The resulting microballoon actuator is extremely large force $(>100 \mathrm{mN})$ and extremely high actuation length $(>1 \mathrm{~mm})$ but, unfortunately, low-step response $(<50 \mathrm{~Hz})$ due to the relatively large air displacement required for actuation. Furthermore, this actuator did not yield results reported by similar studies based on CFD. This is not at all surprising since many CFD results in turbulent regime are not experimentally verifiable due to the lack of physical mechanisms to repeat the simulated scenarios. Fig. 5 shows the actuation height of the balloon actuator versus the drive pressure. Notice at $10 \mathrm{psi}$, the actuation height is about 2-mm out of plane.

The definitive actuator used is a flap-type actuator pneumatically actuated by a silicone membrane underneath [28]. This is 


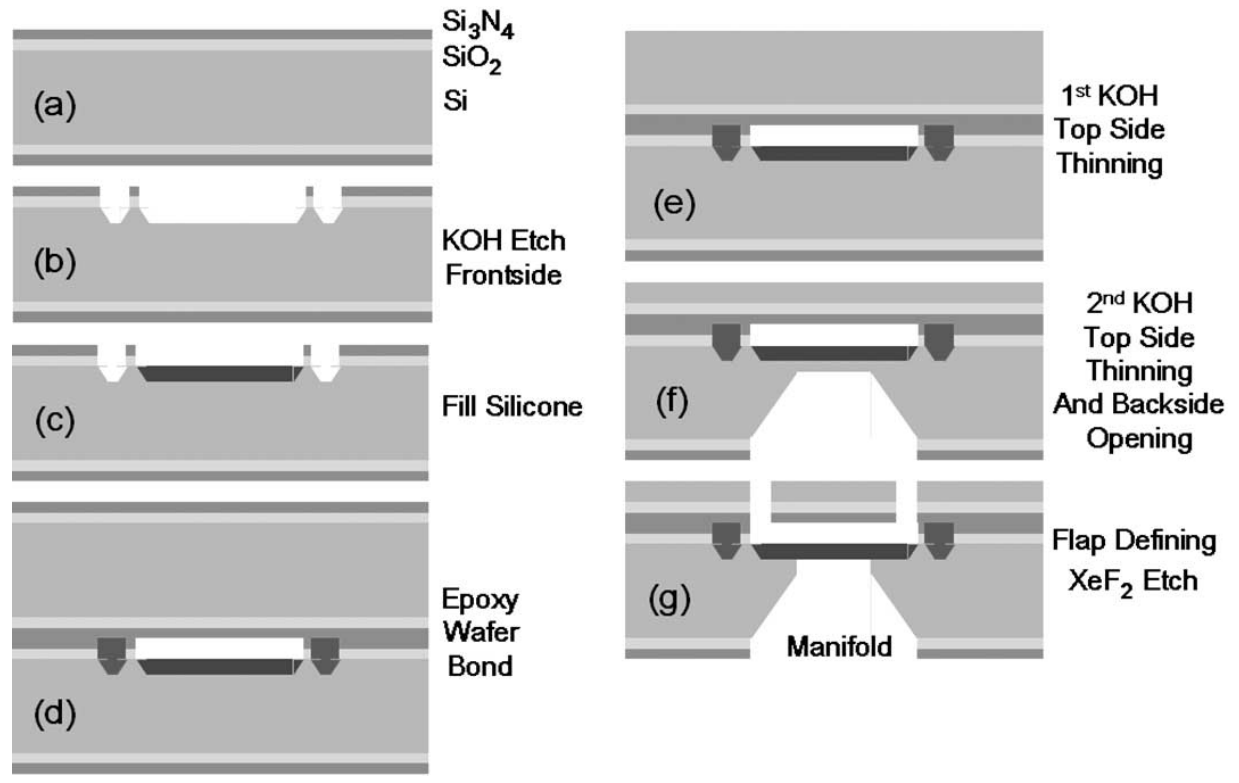

Fig. 6. Process flow of the microflap actuator. (a) Bare silicon wafer coated with silicon dioxide and silicone nitride. (b) Potassium hydroxide (KOH) etch step is used to etch "mold" pits. (c) Silicone is filled in the large pit areas. (d) Wafer bonding with Epoxy glue filling the minor pit areas. (e) First top-side KOH wafer thinning. (f) Bottom-side manifold opening and continuing $\mathrm{KOH}$ etch. (g) Flap shape defining with xenon diflouride $\left(\mathrm{XeF}_{2}\right)$ gas etch and manifold breakthrough etch to the silicone diaphragm.

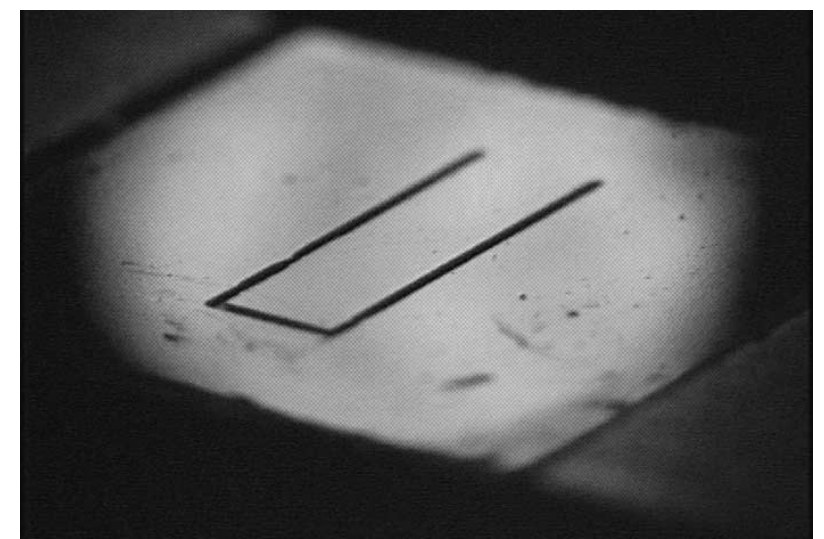

Fig. 7. Definitive microballoon-flap actuator used for the TBL drag reduction experiments. The flap dimensions are $1 \times 3 \mathrm{~mm}$.

basically a combination of both the flap-type actuator with the microballoon actuator. The major difference is that the balloon actuation provides the large force while the flap-type geometries provide the necessary characteristics for turbulent streak interactions. The response time is reduced by decreasing the air displacement used for actuation. The resulting performance is a $\sim 10-\mathrm{mN}$ actuator with $\sim 10$-ms square wave response, $\sim 100-\mu \mathrm{m}$ actuation height, and the ability to actuate and hold at the actuation height on the same cycle. Fig. 6 shows the process flow of the microballoon-flap actuator. Fig. 7 shows the actual microballoon-flap actuator.

\section{Turbulent Wind Tunnel Tests}

Two MEMS shear stress imaging array and the microballoon-flap actuator was then mounted in a two dimensional turbulent wind tunnel (Fig. 8). The sensors are driven by a constant temperature anemometry circuit with an overheat ratio of $12 \%$, which results in the sensor shear stress sensitivity

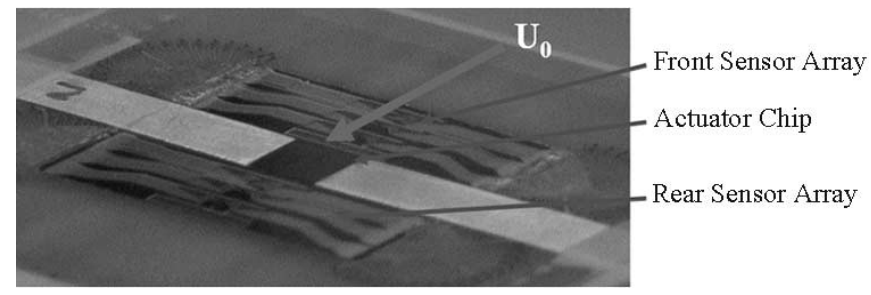

Fig. 8. TBL setup with the microballoon-flap actuator between two shear stress imagers. The front array detects pre-actuation flow while the rear (downstream) array detects the effect of the actuator.

of $\sim 500 \mathrm{mV} / \mathrm{Pa}$ (with $10 \times$ amplifier stage) and a frequency response of $\sim 20 \mathrm{kHz}$. In addition, a single-sensor element on each sensor array chip was driven at a lower overheat ratio $(\sim 1 \%)$ in order to use it as the on-chip temperature sensor for real-time temperature compensation. The dimension of the microballoon-flap actuator used is a 3-mm-long (streamwise) $\times 1$-mm-wide rectangular beam, with a thickness of $\sim 40$ $\mu \mathrm{m}$. Direct, visual measurements have shown that the flap can achieve off-plane deflections of over $130 \mu \mathrm{m}$ at frequencies up to $150 \mathrm{~Hz}$, with a rise time of $\sim 2 \mathrm{~ms}$ and a fall time of $\sim 4 \mathrm{~ms}$. Higher actuation frequencies can be achieved through optimization of the pneumatic components.

The above components are integrated into a control system by flush mounting two sensor arrays, upstream and downstream, from a flap actuator. Experiments are carried out with the system installed onto the wall of a two-dimensional turbulent wind tunnel. The orientation of the system is shown in Fig. 8. At Re $\sim 10 \mathrm{k}$, corresponding to flow velocity of $10 \mathrm{~m} / \mathrm{s}$, time-averaged shear stress reduction of $\sim 4 \%$ downstream of the actuator has been achieved through constant flap actuation at $\sim 100 \mu \mathrm{m}$ and $50 \mathrm{~Hz}$, as shown in Fig. 9. Notice that the time lag between the upstream sensor and the down stream sensor is due to the convection time constant (the time it takes a shear 

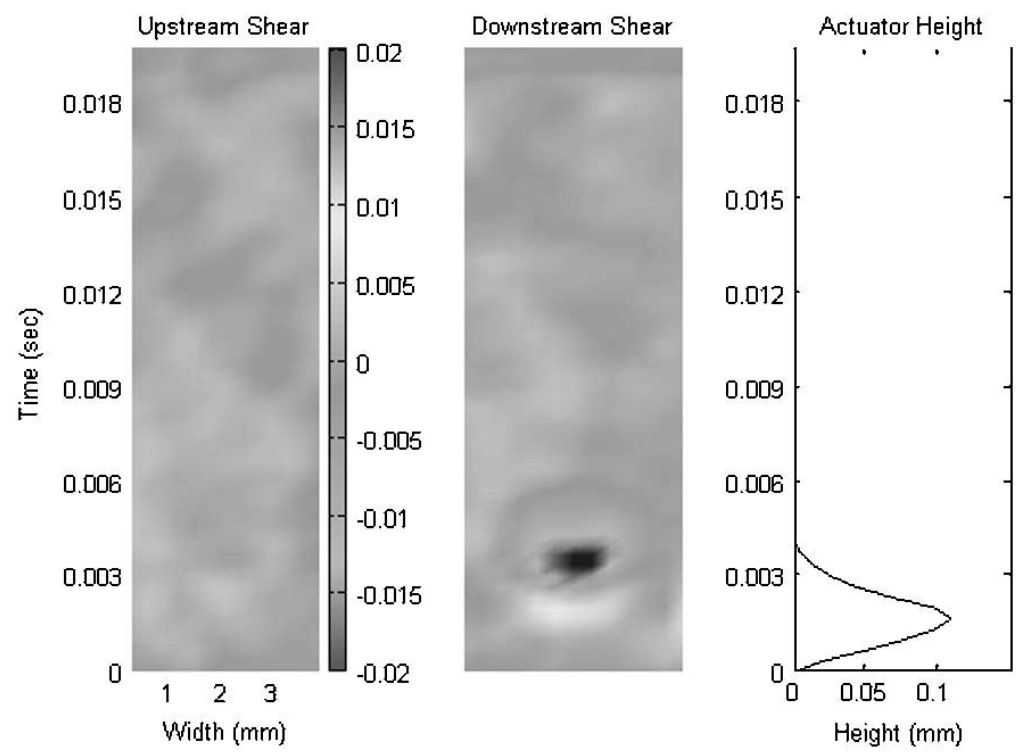

Fig. 9. Ensemble averaged data of the shear stress imager both upstream and downstream of the balloon-flap actuators. Notice the significant dip in the shear stress after the flap actuation. The corresponding actuation height is about $100 \mu \mathrm{m}$.

streak to transport between the two imagers). Shear measurements upstream of the actuator show no change. Furthermore, in offline data processing, it has been found that the actuator can interact with the streak structures and can reduce the peak shear stress of a streak structure by an additional $0.2 \mathrm{~Pa}$, or about $50 \%$ more than the natural decay of streak. A detection algorithm for application of real-time control is currently being developed. For more detailed aspect of the TBL, please refer to [28]. This is the first published result of the TBL experiment data.

\section{Delta Wing Aircraft CONTROL}

\section{A. VSC}

This part of the MEMS macrofluidics control project involves demonstrating the concept of using small actuators $(\sim$ micromillimeter scale) to provide large control forces for a large-scale system $(\sim$ meters/scale). It has been known that the ensuing leading edge vortex flow is extremely sensitive at the separation location of the free shear layer on a swept aerodynamic planform. Contrary to sharp leading edges with the natural boundary condition of separation at the sharp edges, round leading edges have the genesis location of the free shear layer as a complex curved line that runs along the leading edge from the apex to the wing tip. Changing the surface boundary condition in quasisteady state (i.e., expanding surface curvature) near the separation line can cause a global change to the developing vortex. Lee et al. [29] has compiled an excellent comprehensive review on the topic of lift forces of delta wings. Furthermore, it has been shown that instability effects can also manipulate separated vortex flows. Ho et al. [30] has shown that very small forcing amplitudes can control the vortex structures downstream of the mixing layer if the correct subharmonic perturbation of the most energetic frequency is used to manipulate the spreading rate in the vortex evolution.
In general, delta wing leading edges create symmetric pairs of primary and secondary vortices. The controlled separation of the thin boundary layer at the synthesis location of the vortex pairs on a delta wing aircraft will allow the manipulation of pressure field about the aircraft. By using microactuators along the round leading edges to create vortex shifts, a resulting loading change about the aircraft would occur.

It was speculated from the beginning that the boundary layer thickness would be the key to the flap/vortex interaction. Therefore, the length scale of the microactuators needs to be on the order of the boundary layer thickness. The boundary thickness was estimated to be between hundreds of microns to $1-2 \mathrm{~mm}$, depending on the length scale of the aircraft and the Reynolds number. Thus, it was hypothesized that the vortex shifting mechanisms with microactuators can be used to replace or supplement conventional surfaces at high angles of attack where the latter became ineffective due to trailing edge separation of the potential flow.

\section{B. Flexible Shear Stress Sensors for VSC}

It was very clear since the onset of this project that a high sensitivity and robust shear stress sensor is needed in order to detect the sensitive location of the curved separation line. Earlier actuator tests were done in a systematic fashion of eliciting the control forces and moments. This fashion of blind open loop actuation was extremely time consuming and ineffective. For example, the largest rolling moment coefficient created by the MEMS flap actuators similar to the $\mathrm{Ni}_{80} \mathrm{Fe}_{20}$ actuator from the TBL section was found to be only on the order of $\sim 0.001$. The availability of shear stress sensor quickly allowed the authors to target the region near the separation line. Luckily, the rolling moment coefficient was later found to be one order of magnitude higher with the correct actuation location.

However, the sensor technology did not come easily. The first MEMS thermal shear stress sensor as used in the TBL section was fabricated on silicon substrates. The rigid substrate shear 


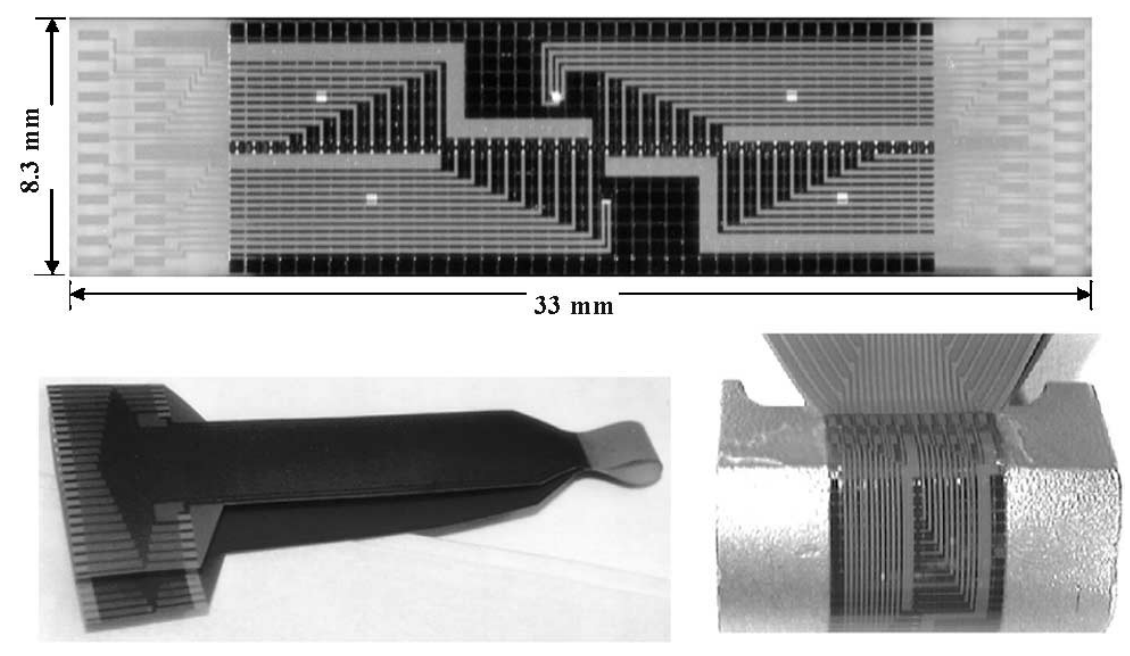

Fig. 10. Latest generation of flexible MEMS shear stress sensor array on Kapton PCB.

stress sensor is not a practical solution for the round leading edge of a delta wing aircraft. Early experiments using rigid silicon substrates required a relentless dedication to measure thousands of leading edge locations by manually rotating and displacing a single sensor along the leading edge radius.

A flexible "skin" solution must be achieved for realistic usage on the curved surfaces of round leading edge delta wings. The flexible skin MEMS shear stress sensor was indeed developed specifically for this project [31], [32]. By using thin polyimide films, a flexible membrane holding each shear stress sensor in a faceted manner (silicon islands) was fabricated. There are roughly four iterations of design for this flexible skin MEMS shear stress sensor and is currently extremely mature. Fig. 10 shows the latest generation of flexible shear stress sensor and the associated flexible Kapton PCB. After "ironing" on the flexible MEMS shear stress skin to the PCB, the PCB is then wrapped on a small section of the leading edge radius. This sensor array contains 36 shear stress sensor elements coated with a thin layer of silicon nitride for maximum robustness in harsh environments.

\section{Flexible Microballoon Actuators for VSC}

Initially, a mechanical actuator using 1-mil stainless steel sheets was used to simulate the MEMS actuators [33], [34]. It should be noted that aerodynamically there is no difference between the stainless steel actuator and flap-type MEMS actuators. A servomotor was used to actuate the stainless steel strips to actuator lengths of $1-5 \mathrm{~mm}$. As with all the subsequent experiments, a $1 / 2^{\prime \prime}$ diameter rod was used as the leading edge of the "flat plate"-type wind tunnel model.

Next, the same $\mathrm{Ni}_{80} \mathrm{Fe}_{20}$ permalloy magnetic flap actuator design as with the TBL experiments was used. It is rather obvious that the MEMS flap-type actuators will not be the solution to this project. MEMS actuators in general has the capability of producing $<100 \mu \mathrm{N}$ [35], which is at least a few orders of magnitude off from the requirement. Also, MEMS flap actuators are not robust enough for high-speed flights at the leading edge of aircraft wings. It has been demonstrated in the wind tunnel that at speeds higher than $30 \mathrm{~m} / \mathrm{s}$, a large percentage of

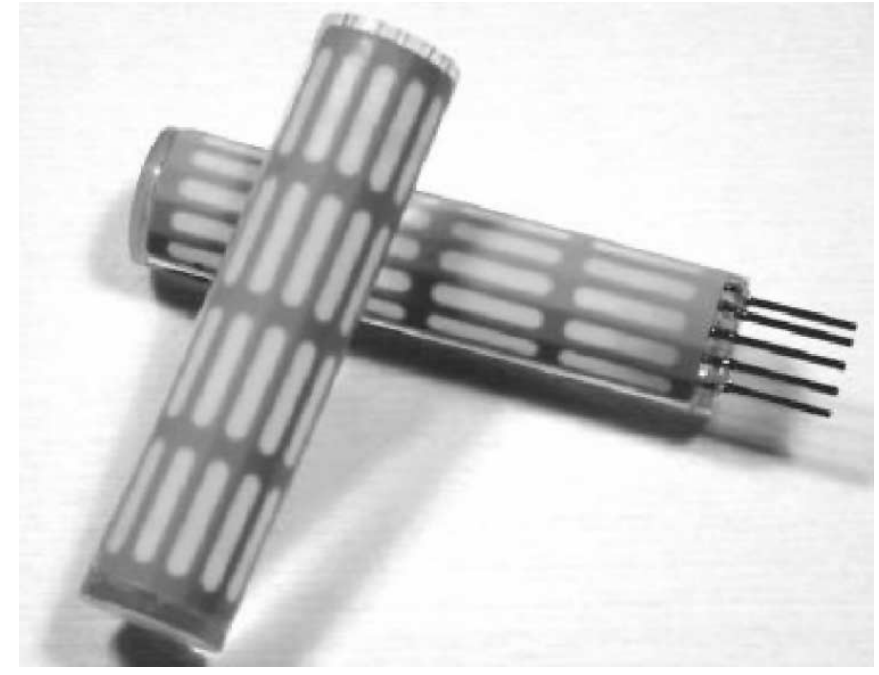

Fig. 11. Flexible balloon actuator arrays mounted on the $1 / 2^{\prime \prime}$ diameter leading edge of the wind tunnel delta wing model.

the MEMS leading edge flap actuators were stripped off by the free stream. By making arrays of the bubble actuators (similar to the single-bubble actuator used for the TBL experiments), finally the MEMS actuator has started to show promise for realistic applications on a real aircraft. The MEMS bubble actuator arrays have gone through two to three generations. The first generation used anisotropically-etched silicon substrates to provide the manifold for gas injection. This type of actuator was designed for ease of fabrication on existing silicon wafer processes. Fig. 4 shows the silicon substrate bubble actuators being inflated at various pressures. However, the rigid substrate is not a practical solution for adoption on aircraft surfaces. The current MEMS balloon actuator is fabricated on metal substrate (brass or copper). The 50-mil copper substrate used on the final version allows a conformal surface to be covered by the flexible balloon actuators. Fig. 11 shows the flexible metal substrate wrapped around a $1 / 2^{\prime \prime}$ leading edge rod used for the wind tunnel model. The bubble actuator technology on flexible metal substrate was then selected as the definitive effectors for this project. Huang $e t$ 


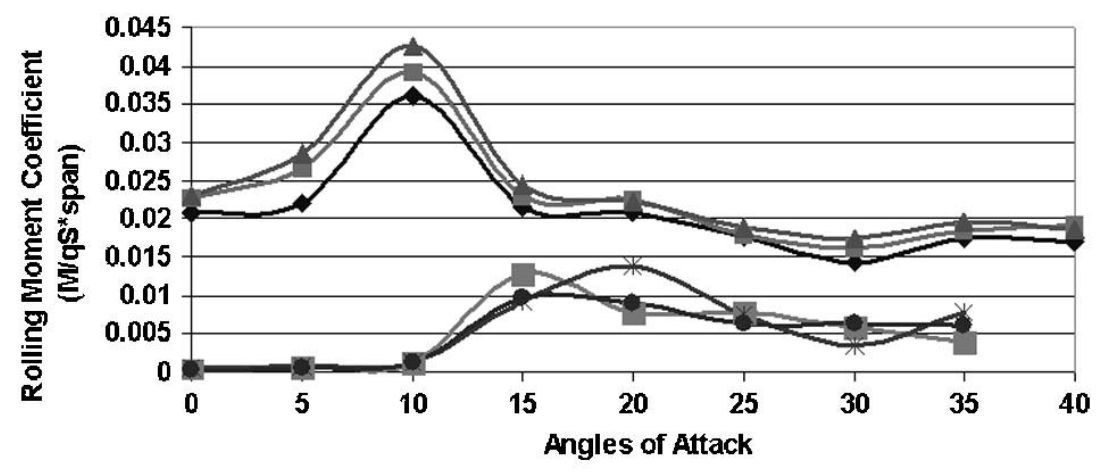

$\rightarrow 10 \mathrm{~m} / \mathrm{s}$ conventional $-15 \mathrm{~m} / \mathrm{s}$ conventional $\rightarrow-20 \mathrm{~m} / \mathrm{s}$ conventional
$-10 \mathrm{~m} / \mathrm{s}$ MEMS $\quad \rightarrow 15 \mathrm{~m} / \mathrm{s}$ MEMS

Fig. 12. MEMS balloon actuator versus conventional ailerons on the same wind tunnel delta wing model. The length scale difference between the two is almost two orders of magnitude.

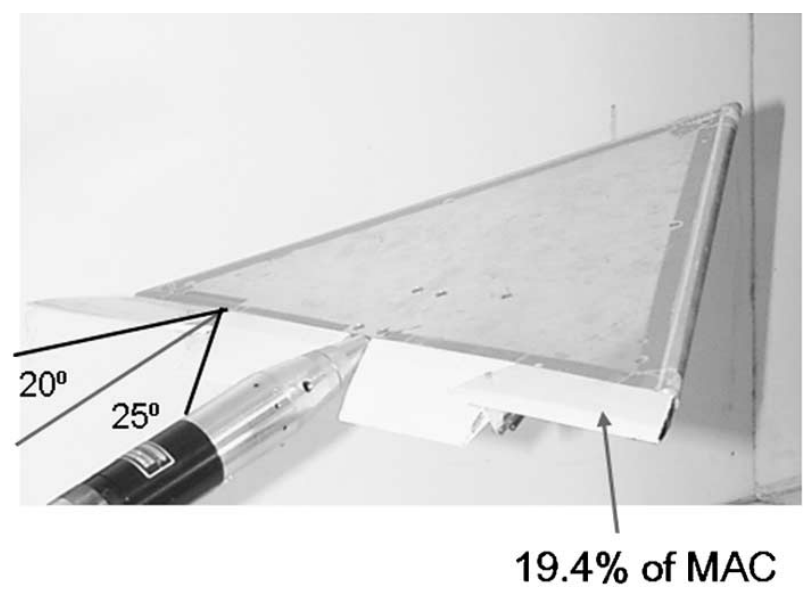

Fig. 13. Conventional aileron actuator used in comparison against the MEMS balloon actuators.

al. [36] described the entire history of the VSC with the authors and detailed development from sensors and actuator developments to UAV-sized aircraft application and finally to transonic flight tests with NASA Dryden's F15B.

\section{VSC Experiments}

A comparison between the bubble actuators and conventional aileron surfaces were performed. The conventional aileron used (outer ailerons, half of the semi-span) has a chord of $19.4 \%$ of the mean aerodynamic chord while the MEMS bubble actuator is only $0.8 \%$ of the mean aerodynamic chord while the generated rolling moment coefficient is only $\sim 50 \%$ less and sufficient for specific flight maneuverings at high angles of attack. Fig. 12 shows the rolling moment comparison between these two types of control surfaces. The aileron deflections used were $+20^{\circ} /-25^{\circ}$ (Fig. 13). It should be noted that the MEMS bubble actuators used were linear; this means that Fig. 12 may not show the maximum possible by the bubble actuators since increasing the spatial resolution of the actuators by segmenting apart the arrays to adapt to the local flow characteristics may yield even higher control forces. This is currently being pursued in conjunction with unsteady delta wing motion in a wind tunnel.

\section{CONCLUSION}

Finally, both the microsensors and actuators required for macrofluidic control are now available, specifically for the use of turbulent boundary layer drag reduction and vortex shifting concept for controlling a full scale aircraft. Further research in the applied fluid dynamics with these important tools will yield significant advances in the art of fluid dynamic and aerodynamic engineering in the near future. In terms of the TBL drag reduction applications on a practical aero-vehicle, a conformable actuator with flap geometries need to be invented. Unfortunately, as noted before, the bubble actuator without the flap edge was found to be ineffective for TBL control. Thus, the flexible bubble actuator arrays cannot be used for TBL control. Current work includes creating a patterned silicone membrane to facilitate fabrication of the bubble-flap actuator on a flexible substrate.

\section{ACKNOWLEDGMENT}

The authors would like to thank all of those involved in the TBL and VSC experiments (numbering over 20 researchers) due to the long-term work of these two projects, spanning over almost a decade.

\section{REFERENCES}

[1] [Online]. Available: http://www.analogdevices.com

[2] [Online]. Available: http://www.honeywell.com

[3] [Online]. Available: http://www.msiusa.com

[4] [Online]. Available: http://www.hp.com

[5] [Online]. Available: http://www.ti.com

[6] J. J. Bertin, Aerodynamics for Engineers, 4th ed. Upper Saddle River, NJ: Prentice-Hall, 2002, pp. 109-151.

[7] S. J. Kline, W. C. Reynolds, F. A. Schraub, and P. W. Runstadler, "The structure of turbulent boundary layers," J. Fluid Mech., vol. 30, no. 4, pp. 741-773, 1967.

[8] J. H. Hool, "Measurement of skin friction using surface tubes," Aircraft Eng., vol. 28, p. 52, 1956.

[9] L. F. East, "Measurement of Skin Friction at Low Subsonic Speeds by Razor-Blade Technique," Aero. Res. Council., 1966.

[10] R. J. Goldstein, Fluid Mechanics Measurement. New York: Hemisphere, 1983, p. 61.

[11] J. H. Preston, "The determination of turbulent skin friction by means of pitot tubes," J. R. Aero. Soc., vol. 58, pp. 109-121, 1953.

[12] M. A. Schmidt, R. T. Howe, S. D. Senturia, and J. H. Haritonidis, "Design and calibration of a microfabricated floating-element shear-stress sensor," IEEE Trans. Electron Devices, vol. 35, pp. 750-757, June 1998. 
[13] J. Shajii, K. Y. Ng, and M. A. Schmidt, "A microfabricated floatingelement shear stress sensor using wafer-bonding technology," ASME J. Microelectromech. Syst., vol. 1, pp. 89-94, 1992.

[14] T. Pan, D. Hyman, M. Mehregany, E. Reshotko, and B. Willis, "Calibration of microfabricated shear stress sensor," in Dig. Tech. Papers Transducers, 1995 , pp. 443-446.

[15] M. Mehregany and C. Bang, "MEMS for smart structures," in Proc. Conf. Smart Structures and Materials, 1995, pp. 105-114.

[16] A. Padmanabhan, H. D. Goldberg, K. S. Breuer, and M. A. Schmidt, "A silicon micromachined floating-element shear-stress sensor with optical position sensing by photodiodes," Dig. Tech. Papers Transducers, pp. 436-439, 1995.

[17] J. B. Huang, F. K. Jiang, Y. C. Tai, and C. M. Ho, "A micro-electro-mechanical-system-based thermal shear stress sensor with self-frequency compensation," Meas. Sci. Technol., vol. 10, pp. 687-696, 1999.

[18] C. Liu, J. Huang, A. Zhu, F. Jiang, S. Tung, Y. C. Tai, and C. M. Ho, "A micromachined flow shear stress sensor based on thermal transfer principles," ASME J. Microelectromech. Syst., 1999.

[19] F. Jiang, Y. C. Tai, B. Gupta, R. Goodman, S. Tung, J. B. Huang, and C. M. Ho, "A surface-micromachined shear stress imager," in Proc. IEEE Conf. Microelectromechanical Systems, 1996, pp. 332-335.

[20] Y. Xu, F. Jiang, Q. Lin, J. Clendenen, S. Tung, and Y. C. Tai, "Underwater shear-stress sensor," in Proc. IEEE Conf. Microelectromechanical Systems, Las Vegas, NV, 2002, pp. 340-343.

[21] R. L. Simpson, M. Ghodbane, and B. E. McGrath, "Surface pressure fluctuations in a separating turbulent boundary layer," J. Fluid. Mech., vol. 177, pp. 167-186, 1987.

[22] J. D. Ruedi, H. Nagib, J. Österlund, and P. A. Monkewitz, "Evaluation of three techniques for wall-shear measurements in three-dimensional flows," Exper. Fluids, vol. 35, pp. 389-396, 2003.

[23] J. M. Österlund, B. Lindgren, and A. V. Johansson, "Flow structures in zero pressure-gradient turbulent boundary layers at high reynolds numbers," Eur. J. Mech. B, vol. 22, pp. 379-390, 2003.

[24] C. Liu, T. Tsao, Y. C. Tai, T. S. Leu, C. M. Ho, W. Tang, and D. Miu, "Micromachined permalloy magnetic actuator for delta-wing control," in Proc. IEEE Conf. Microelectromechanical Systems, 1995, pp. 332-335.

[25] C. Liu, T. Tsao, G. B. Lee, T. S. Leu, Y. C. Tai, and C. M. Ho, "Out-of-plane magnetic actuators with electroplated permalloy for fluid dynamics control," Sens. Actuators A, vol. 78, no. 2-3, 1994.

[26] C. Liu, T. Tsao, Y. C. Tai, and C. M. Ho, "Surface micro-machined magnetic actuators," in IEEE Workshop Microelectromechanical Systems, Oiso, Japan, 1994, pp. 57-62.

[27] C. Grosjean, G. B. Lee, W. Hong, Y. C. Tai, and C. M. Ho, "Micro balloon actuators for aerodynamic control," in IEEE Workshop Microelectromechanical Systems, Heidelberg, Germany, 1998, pp. 166-171.

[28] J. Lew, A. Huang, F. Jiang, Y. C. Tai, and C. M. Ho, "Surface shear stress reduction with MEMS sensors/actuators in turbulent boundary layers," in Proc. 42nd AIAA Aerospace Sciences Meeting, Reno, NV, 2004

[29] M. Lee and C. M. Ho, "Lift forces of delta wings," in Appl. Mech. Rev., 1990, vol. 43

[30] C. M. Ho and L. S. Huang, "Subharmonics and vortex merging in mixing layers," J. Fluid Mech., vol. 119, pp. 443-473, 1982.

[31] F. Jiang, Y. Xu, T. Weng, Z. Han, Y. C. Tai, A. Huang, C. M. Ho, and S. Newbern, "Flexible sensor skin for aerodynamics applications," in Proc IEEE Conf. Microelectromechanical Systems, 2000, pp. 465-470.

[32] A. Huang, C. M. Ho, F. Jiang, and Y. C. Tai, "MEMS transducers for aerodynamics-A paradigm shift," in Proc. 38nd AIAA Aerospace Sciences Meeting, Reno, NV, 2000.

[33] G. B. Lee, "Control of a Delta-Wing Aircraft by Using Micromachined Sensors and Acutators," Ph.D. dissertation, Dept. Mech. Aerosp. Eng., Univ. California, Los Angeles, CA, 1998.

[34] G. B. Lee and C. M. Ho, "Roll motion control of a delta wing by LE actuators," in Proc. Bull. Amer. Phys. Soc., 49th Annu. Meeting Division of Fluid Dynamics, 1996.

[35] G. T. A. Kovac, Micromachined Transducers Sourcebook. New York: McGraw-Hill, 1998.
[36] A. Huang, C. Folk, C. Silva, B. Christensen, Y. F. Chen, G. B. Lee, M Chen, S. Newbern, F. Jiang, C. Grosjean, C. M. Ho, and Y. C. Tai, "Application of MEMS devices to delta wing aircraft: From concept development to transonic flight test," in Proc. 39th AIAA Aerospace Sciences Meeting, Reno, NV, 2001

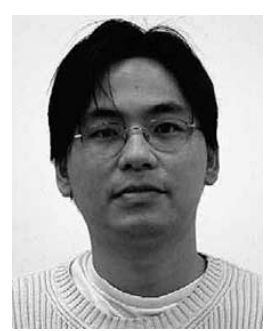

Adam Huang received the B.S. degree in aerospace engineering from the University of California, Los Angeles (UCLA), in 1998. He is currently pursuing the Ph.D. degree at UCLA under the guidance of Dr. C.-M. Ho.

His current research topics include MEMS devices for aeronautical engineering and combustion.

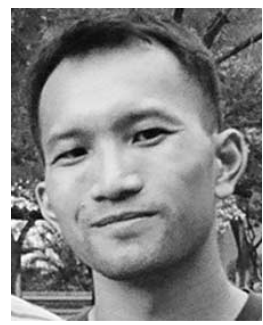

James Lew received the B.S. degree in mechanical engineering from the University of California, Berkeley, in 1994 and the M.S. and Ph.D. degrees in mechanical engineering from the University of California, Los Angeles, under the guidance of Dr. C.-M. Ho, in 1997 and 2004, respectively.

His research topics include turbulence and fluidic control.

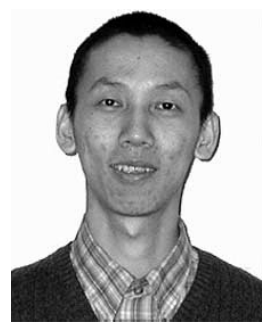

Yong Xu received the B.S. degree in electronics engineering from Tsinghua University, Beijing, China, in 1997 and the M.S. and Ph.D. degrees in electrical engineering from the California Institute of Technology, Pasadena, in 1998 and 2002, respectively.

$\mathrm{He}$ is currently an Assistant Professor in the Department of Electrical and Computer Engineering at Wayne State University, Detroit, MI. His research interests include MEMS smart skin technology, bioMEMS, sensory systems for harsh environments, novel packaging technology, and nanotechnology.

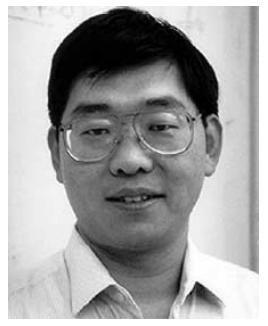

Yu-Chong Tai received the B.S. degree from the National Taiwan University, Taipei, Taiwan, R.O.C. and the M.S. and Ph.D. degrees in electrical engineering from the University of California, Berkeley (UCB), in 1986 and 1989, respectively.

After UCB, he joined the Faculty of Electrical Engineering, California Institute of Technology (Caltech), Pasadena, and built the Caltech $\mathrm{Mi}$ cromachining Laboratory. $\mathrm{He}$ is currently a Full Professor of electrical engineering at Caltech. His research interests include flexible MEMS, integrated microfluidics, neuroprobes and chips, optical MEMS, and biochemical sensors.

Dr. Tai has received several awards, including the IBM Fellowship, the Best Thesis Award, the Presidential Young Investigator (PYI) Award, and the David and Lucile Packard Fellowship. 


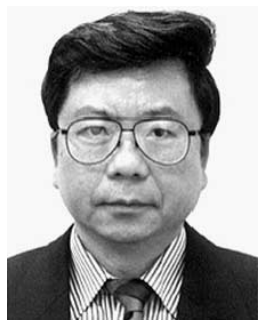

Chih-Ming Ho (M'00) received the B.S. degree from the Mechanical Engineering Department, National Taiwan University, Taipei, Taiwan, R.O.C., and the Ph.D. degree from The John Hopkins University, Baltimore, MD.

He currently serves as University of California, Los Angeles (UCLA), the Associate Vice Chancellor for Research and holds the Ben Rich-Lockheed Martin Chair Professor in School of Engineering, UCLA. He is the Director of Institute for Cell Mimetic Space Exploration, UCLA. He has published over 220 papers and holds seven patents. He is known for his works on micro/nano-fluidics, bio/nanotechnology, and turbulence.

Dr. Ho is a Member of the American Society of Mechanical Engineers (ASME). He was elected a Fellow of the American Physical Society and a Fellow of the American Institute of Aeronautics and Astronautics for his contributions in a wide spectrum of technical areas. In 1997, he was inducted as a member of the National Academy of Engineering and, in 1998, he was elected as an Academician of Academia Sinica. 